Mal J Nutr 27(3): 411-420, 2021

\title{
Relationship between maternal characteristics and stunting in children aged 0 to 23 months in the Philippines
}

\author{
Alea Paula V. Hinojosa ${ }^{1}$, Nancy A. Tandang ${ }^{1} \&$ Divine Grace C. Domingo ${ }^{2^{*}}$ \\ ${ }^{1}$ Institute of Statistics, College of Arts and Sciences, University of the Philippines Los \\ Banos; ${ }^{2}$ Institute of Human Nutrition and Food, College of Human Ecology, University \\ of the Philippines Los Banos
}

\begin{abstract}
Introduction: Stunting is the most prevalent form of child malnutrition worldwide, and is the best overall indicator of growth. This study aimed to determine the relationship between maternal characteristics and nutritional status of children aged 0-23 months based on their length/height-for-age. Method: The study used data from the 2015 Updating Survey conducted by the Philippines' Food and Nutrition Research Institute - Department of Science and Technology. It employed a stratified multi-stage sampling technique covering all regions in the Philippines. Mothers $(n=5,254)$ of sampled children 0-23 months were the respondents of the Maternal Health and Nutrition survey. The length-for-age $z$-scores (LAZ) of children and maternal characteristics were analysed. Rao-Scott Chi-square test was used for association analysis. Logistic regression was performed for model fitting. Results: A quarter $(25.2 \%)$ of children aged $0-23$ months in the Philippines at the time of the survey were stunted, with the highest prevalence $(36.2 \%)$ observed among 12-23 months. Children being stunted or normal in height was associated with prenatal services, maternal nutritional status, education, and duration of lactation. Maternal education (OR: 0.39; $p=0.012$ ), age-appropriate breastfeeding (OR: 0.63; $p=0.042$ ), and prenatal services like tetanus toxoid vaccination (OR: $0.67 ; p=0.011$ ) and ultrasound (OR: $0.71 ; p=0.025$ ) lowered the likelihood of a child being stunted. Conclusion: It is recommended to strengthen and intensify service delivery among pregnant and lactating women because of the implication of maternal factors to the length-for-age status of children 0-23 months.
\end{abstract}

Keywords: stunting, maternal factors, length-for-age, education, young children

\section{INTRODUCTION}

Stunting is a form of chronic undernutrition and is used to describe populations of children who are short for their age (UNICEF, 2019). According to de Onis and Branca (2016), childhood stunting is the best overall indicator of well-being in children. Irreversible consequences of stunting under the age of 24 months include compromised motor skills and cognitive function, which may insinuate poor academic performance in the future (UNICEF, 2007). Stunting is the most prevalent form of child malnutrition worldwide, where one out of three children aged under 5 years or 149 million children aged under 5 years are affected (UNICEF,

\footnotetext{
*Corresponding author: Divine Grace C. Domingo

Institute of Human Nutrition and Food, College of Human Ecology,

University of the Philippines Los Banos

Tel: (+63)091577902235; Fax: (+63)(049)536-2445; E-mail: dcdomingo2g@up.edu.ph

doi: https://doi.org/10.31246/mjn-2021-0010
} 
2019). In the Philippines, about 33\% or 3.6 million children aged 0-60 months were stunted in 2015, making the country ranked $9^{\text {th }}$ in terms of countries with the highest burden of stunting (DOST-FNRI, 2015). Among all the age groups, the highest rate of stunting was recorded among children between the ages of 12-23 months with a prevalence of $36 \%$. Moreover, there has been little improvement in the percentage of stunted children since 2003 when the prevalence of stunting decreased to $34 \%$ from the previously recorded $45 \%$ in 1989. The observed innate relationship between mother and child made past researches like Babatunde et al. (2011), and Kandpal and McNamara (2009), use maternal characteristics as possible factors in predicting child nutrition, since young children are more dependent on their caretakers, which oftentimes are their mothers.

Improvement in child nutrition has long been associated with human capital investment. Healthier children are bound to have lesser chances of suffering from premature death compared to malnourished children. In addition, stunting among young children, in particular, is known to affect the mental capacity of children, as well as their cognitive skills (UNICEF, 2007). The psychological function was also found to be negatively affected amongst adolescents that have a history of stunting before they were two years old (Walker et al., 2007). Anxiety, depression, and low self-esteem were more prevalent amongst those that suffered from stunting before the age of two compared to those with no history of stunting (Walker et al., 2007). The study by Boah et al. (2019) in Ghana showed that food intake, birth weight, history of diseases, and sex are some of the main factors that are related to malnutrition among young children. The study also mentioned that maternal nutrition can affect foetal growth because there are some intrauterine growth limitations commonly observed among underweight mothers. Socioeconomic factors have also been observed to be related to young child malnutrition. Based on the factors mentioned, there is high maternal influence observed in the nutrition of infants and young children- especially since children aged 0-23 months are highly dependent on their mothers. Thus, this study aimed to determine the relationship between maternal characteristics and nutritional status of children aged 0-23 months based on their height-for-age anthropometric indicator. Besides that, the likelihood of stunting among children aged 0-23 months using maternal characteristics as predictors was identified using an estimated model.

The results of this study can be a basis for policy makers in the Philippines in its design of national interventions to address stunting, specifically focusing on maternal factors that relate to stunting within the first two years of life. Stunting in children is known to have grave irreversible effects on the child's growth and development, therefore nutrition interventions and policies on stunting must recognise the importance of addressing maternal factors that might have an impact on improving the malnutrition situation. Future studies on stunting or malnutrition among infants and young children may use the results of this study as a reference to achieve a deeper understanding of child nutrition.

This study used the survey data on children under two years of age in the Philippines. Any conclusions that could be made from this study will only reflect children of that age group in the country. Since the data used were from the 2015 Updating of the Nutritional Status of Filipino Children and Other Population Groups survey conducted by the Department of Science and Technology-Food and Nutrition Research Institute (DOST-FNRI), more recent data could reveal different results from 
this study. Any factors related to infant nutrition other than that of maternal characteristics were not included nor reflected in the study.

\section{MATERIALS AND METHODS}

\section{Data source}

The study used the Maternal Health and Nutrition and Infant and Young Child Feeding (MHN-IYCF) Surveys, SocioEconomic Survey, and Anthropometric Survey data from the 2015 Updating Survey conducted by the Food and Nutrition Research Institute Department of Science and Technology (FNRI-DOST). The survey used the Master Sample (MS) developed by the Philippine Statistics Authority (PSA) where the country's 17 administrative regions served as the domains. Stratified multi-stage sampling was employed to cover all regions and provinces in the country. The first stage was the selection of the primary sampling units (PSUs), which were barangays with at least 500 households. Following that was the selection of enumeration areas (EAs) or barangays with 150-200 households, and then the selection of households from EAs, which served as sampling units (SUs). Respondents for the MHN survey were mothers with their youngest biological child aged between 0-36 months, while the IYCF survey considered households with children aged between 0-23 months (FNRI-DOST, 2016). This study considered children under 2 years of age with no missing information on their measurements and their mother's characteristics, yielding a total of 5,254 sampled children aged 0-23 months.

\section{Variables of interest}

The dependent variable considered in the study was the nutritional status of children aged $0-23$ months based on their length-for-age $z$-scores (LAZ), classified as stunted, normal, and tall. LAZs were computed using the World
Health Organization (WHO) Anthro Survey Analyser. Children with $z$-scores of <-2 standard deviation (SD) were classified as stunted, those with $-2 S D$ to $+2 S D$ were considered as normal in height, and those with $z$-scores of $>+2 S D$ were identified as tall. Moreover, the magnitude and severity of malnutrition in the country in terms of stunting was assessed as low, medium, high, and very high with the prevalence of stunting as $<20 \%, 20-29 \%, 30-39 \%$, and $\geq 40 \%$, respectively (WHO/UNICEF, 2019). The maternal characteristics used in the study were clustered into four groups. The first group comprised of sociodemographic information about the mothers such as ethnicity, educational attainment, employment status, and occupation. The second group was about the fertility and health of the mothers, which included nutritional status, vitamin intake, and all the physiological status of mothers. The third group composed of those variables that determined pregnancy practices such as prenatal care/lack of prenatal care, taking/failure to take pregnancy supplements, and counselling/ lack of counselling during pregnancy. The last group comprised of maternal knowledge and practices like breastfeeding and complementary feeding.

\section{Data collection}

The length of children younger than two years old and the height of those two years and older were measured using a medical plastic infantometer and stadiometer, respectively. The length/ height measurements were recorded to the nearest $0.1 \mathrm{~cm}$, adhering to standard procedures. Calibration of the instruments was done using standard procedures. Meanwhile, data on maternal characteristics were generated through personal interviews among mothers or primary caregivers of the 0-23 month-old children in sampled households using an electronic data 
collection system (e-DCS) (FNRI-DOST, 2016).

\section{Ethical review}

The 2015 Updating Survey received approval from the FNRI Institutional Ethics and Review Committee. Written consent was obtained from the subjects through the mother or guardian for the conduct of the survey and measurements. Moreover, all the questionnaires used in the survey were pretested and approved by the PSA (FNRI-DOST, 2016).

\section{Data analyses}

The subjects in this study were described by constructing weighted percentage distributions. Likewise, the nutritional status of children 0-23 months based on the length-for-age indicator was presented by constructing a weighted percentage distribution. To test whether the stunting condition of children was associated with maternal characteristics, the Rao-Scott Chi-square test was used. To measure the degree of association between anthropometric variables and maternal characteristics, the Cramer's $V$ coefficient was computed, which ranged from 0 to 1 only. A value between 0 and 0.1 indicated a weak association between the variables, while a value between 0.1 and 0.3 referred to a moderate association, and a coefficient of $>0.3$ denoted a strong association between the variables (Akoglu, 2018). Factors found with significant association to the stunting condition of a child were further subjected to model fitting using the binary logistic regression analysis. The nutritional status based on LAZ, which served as the dependent variable, was set to 0 for the normal category and 1 for stunting. The assumption that each child belonged to one of the two mutually exclusive categories $(0$ or 1) was held. The normal category $(Y=0)$ served as the reference category. The tallness of a child using LAZ is usually not a problem unless there is the presence of uncommon endocrine disorders that cause excess growth hormones (WHO, 2008). There was no information about endocrine disorders in the 2015 Updating Survey, therefore, a separate category for tallness was not considered in this study.

Before model fitting, the data were divided into two parts: one part to serve as a training dataset and the other as a test dataset. The training dataset, which was $70 \%$ of the original dataset, was used to fit the models for each anthropometric indicator. The remaining $30 \%$ of the original data - the test data, was used to assess the predictive ability of the models. The $70-30$ ratio of the training and test dataset is one of the most common ways of partitioning datasets from empirical studies and is sometimes considered the rule of thumb (Ng, 2017). Division of the original dataset was necessary to avoid overfitting, which can occur when the generated model becomes too specific for a dataset that it becomes unusable for other datasets (Gindro, 2020). The generated models were assessed based on the corresponding values on the diagnostic tests: sensitivity, specificity, and overall classification rate (Altman \& Bland, 1994). Sensitivity (true positive rate) provides the proportion of correctly classified stunted children aged 0-23 months. The higher the value of sensitivity, the more likely the model could be able to predict stunted children. Alternatively, specificity (true negative) provides the proportion of correctly classified children with normal nutritional status. Higher specificity values imply fewer chances of having false positives, meaning the model is able to detect normal children efficiently. Meanwhile, the overall classification rate or accuracy provides the proportion of correctly classified observations (true positives and true negatives). Likewise, the Receiver Operating Characteristic (ROC) curve graphically shows the tradeoff between sensitivity and specificity (Zweig \& Campbell, 1993). The area under the ROC curve (AUC) shows how 
well the estimated model is. In addition, model tests such as the HosmerLemeshow test were applied to show more evidence that the latest or current model has a better fit compared to models with less predictors or the intercept-only model (Fagerland \& Hosmer, 2012). The null hypothesis tested by the model was that the current model is more adequate compared to previous models, therefore, a high $\mathrm{p}$-value from the test would give support to the current model.

\section{RESULTS}

About a quarter (25.2\%) of children aged $0-23$ months at the time of the survey were stunted. The stunted male children outnumbered stunted females with an estimated rate of $28.7 \%$ and $21.6 \%$, respectively. The largest portion of stunted children was registered by those aged between 12 and 23 months, accounting for $36.2 \%$ of the children population - implying that stunting was highly prevalent in this age group, particularly for males with a higher prevalence rate of $40.7 \%$ as compared to females with $31.7 \%$. On the other hand, children aged 0-5 months and 6-11 months both registered low magnitude and severity of stunting with a prevalence rate of $12.1 \%$ and $16.4 \%$, respectively (Table 1).

Among the several maternal characteristics considered in the survey, Table 2 presents those found to be significantly associated with the stunting status of children aged 0-23 months at the $5 \%$ level of significance. In particular, being stunted or normal in height was weakly associated with the practice of post-natal check-up, taking an ultrasound, tetanus toxoid vaccine prenatal services, taking folic acid, and single vitamin and multivitamins during pregnancy, diabetic complications during pregnancy, nutritional status of the mother, and highest educational attainment. Meanwhile, the duration of lactation of a mother was moderately associated with the stunting of children aged 0-23 months. Furthermore, based on the model fitting, highest educational attainment, months of lactation, age-appropriate breastfeeding, having tetanus toxoid vaccination and ultrasound during pregnancy, and trimester of pregnancy were found to be significant influencing factors for a child aged 0 to 23 months being stunted at the $5 \%$ level of significance (Table 3 ). The odds of a child being stunted was $61 \%$ lower if the mother had attained at least a college level of education than if the mother had no formal schooling. Also, whether a mother was lactating or not at the time of the survey was found to be a significant influencing factor for a child being stunted. It was estimated that the odds of a child being stunted was $42 \%$ lower if a mother had been lactating between 1 to 6 months compared to a mother that was not lactating. However, the likelihood to be stunted was 2.64 times higher if the mother was lactating for more than a year compared to a mother that was not lactating. In relation to lactation, it was found in this study that age-appropriate breastfeeding also lowered the odds of a child being stunted

Table 1. Weighted percentage distribution of children aged 0-23 months based on LAZ indicator, by age group and sex in the Philippines

\begin{tabular}{|c|c|c|c|c|c|c|c|c|c|}
\hline \multirow{2}{*}{$\begin{array}{c}\text { Age } \\
\text { group } \\
\text { (month) }\end{array}$} & \multicolumn{3}{|c|}{ Male } & \multicolumn{3}{|c|}{ Female } & \multicolumn{3}{|c|}{ Both sexes } \\
\hline & $\begin{array}{c}\text { Stunted } \\
\%\end{array}$ & $\begin{array}{c}\text { Normal } \\
\%\end{array}$ & $\begin{array}{c}\text { Tall } \\
\%\end{array}$ & $\begin{array}{c}\text { Stunted } \\
\%\end{array}$ & $\begin{array}{c}\text { Normal } \\
\%\end{array}$ & $\begin{array}{c}\text { Tall } \\
\%\end{array}$ & $\begin{array}{c}\text { Stunted } \\
\%\end{array}$ & $\begin{array}{c}\text { Normal } \\
\%\end{array}$ & $\begin{array}{c}\text { Tall } \\
\%\end{array}$ \\
\hline $00-05$ & 14.3 & 81.7 & 4.0 & 11.0 & 82.5 & 6.5 & 12.7 & 82.0 & 5.3 \\
\hline 06-11 & 21.1 & 75.7 & 3.2 & 13.2 & 83.5 & 3.3 & 17.3 & 79.4 & 3.3 \\
\hline $12-23$ & 40.7 & 56.3 & 3.0 & 31.7 & 65.6 & 2.7 & 36.2 & 61.0 & 2.8 \\
\hline
\end{tabular}


by $37 \%$. Meanwhile, a child whose mother was in her third trimester of pregnancy at the time of the survey was more likely to be stunted by almost three times (2.95) compared to a child whose mother was not pregnant. Pregnancy practices of mothers also contributed to child nutrition. A decrease of 33\% in the chance of a child being stunted was observed if the mother had availed tetanus toxoid vaccination during her pregnancy. Lastly, the mother having an ultrasound during pregnancy also decreased the chance of a child being stunted by $29 \%$. Hosmer and Lemeshow's goodness-of-fit test signified that the estimated model fitted the data well $(p=0.143)$. The generated ROC with an

Table 2. Association between maternal characteristics and stunting status of Filipino children aged 0-23 months

\begin{tabular}{lccc}
\hline \multicolumn{1}{c}{ Maternal Characteristics } & $\begin{array}{c}\text { Chi-square } \\
\text { test statistic }\end{array}$ & $\begin{array}{c}\text { Cramer's } V \\
\text { coefficient }\end{array}$ & p-value* \\
\hline Months of lactation & 61.19 & 0.107 & $<0.001$ \\
Practice of post-natal check-up & 46.17 & 0.094 & $<0.001$ \\
Highest educational attainment & 39.9 & 0.088 & $<0.001$ \\
Trimester of pregnancy & 39.37 & 0.086 & $<0.001$ \\
Prenatal service: ultrasound & 28.64 & 0.075 & $<0.001$ \\
Currently taking vitamins & 24.73 & 0.073 & $<0.001$ \\
Pregnancy supplement: single vitamin/mineral & 17.21 & 0.061 & $<0.001$ \\
Nutritional status & 15.33 & 0.054 & 0.003 \\
Prenatal service: tetanus toxoid vaccine & 12.58 & 0.050 & 0.001 \\
Pregnancy supplement: folic acid & 8.30 & 0.042 & 0.010 \\
Complications in pregnancy due to diabetes & 3.24 & 0.040 & 0.005 \\
Knowledge of child vaccine & 5.27 & 0.032 & 0.023 \\
\hline
\end{tabular}

*significant at $p \leq 0.05$

Table 3. Estimated logistic regression model for stunting among Filipino children aged 0-23 months

\begin{tabular}{|c|c|c|c|c|}
\hline Maternal characteristics & Coefficient & Standard error & Odds ratio & $p$-value* \\
\hline \multicolumn{5}{|l|}{ Education $^{\dagger}$} \\
\hline At least college level & -0.941 & 0.394 & 0.39 & 0.012 \\
\hline \multicolumn{5}{|c|}{ Current months of lactation ${ }^{\ddagger}$} \\
\hline $1-6$ months & -0.545 & 0.276 & 0.58 & 0.049 \\
\hline Over 1 year & 0.969 & 0.264 & 2.64 & $<0.001$ \\
\hline \multicolumn{5}{|c|}{ Age appropriate breastfeeding $\$$} \\
\hline Yes & -0.464 & 0.228 & 0.63 & 0.042 \\
\hline \multicolumn{5}{|l|}{ Trimester of pregnancy $₫$} \\
\hline Third & 1.083 & 0.385 & 2.95 & 0.005 \\
\hline \multicolumn{5}{|c|}{ Prenatal service: tetanus toxoid vaccine ${ }^{\dagger \dagger}$} \\
\hline Availed & -0.405 & 0.160 & 0.67 & 0.011 \\
\hline \multicolumn{5}{|c|}{ Prenatal service: ultrasound ${ }^{\dagger \dagger}$} \\
\hline Availed & -0.342 & 0.152 & 0.71 & 0.025 \\
\hline Constant & -1.220 & 0.393 & 0.30 & 0.002 \\
\hline
\end{tabular}

*significant at $p \leq 0.05$

Base category:

${ }^{\dagger}$ No formal schooling, ${ }^{\ddagger}$ Not lactating, ${ }^{\S}$ No, ${ }^{\circledR}$ Not pregnant, ${ }^{\dagger \dagger}$ Did not avail 
AUC of 0.66 revealed that the generated model has low discriminatory power over stunted and normal children. However, the overall accuracy rate of the model was $70.10 \%$ - fair enough for a model (Figure 1). Moreover, specificity and sensitivity tests showed that $55.24 \%$ of the stunted children were correctly classified, while $71.53 \%$ of the normal children were properly classified. The accuracy tests revealed that the model can discriminate stunted with normal children.

Figure 1. ROC curve generated using the model for LAZ indicator

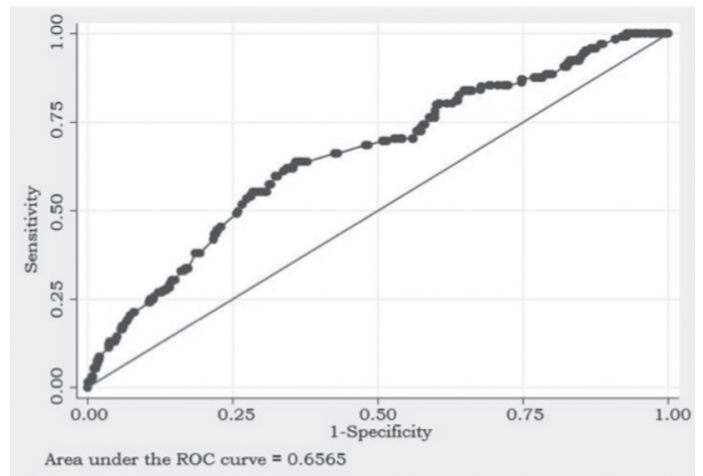

\section{DISCUSSION}

The stunting condition of children aged 0-23 months in the Philippines can be classified in the medium magnitude and severity, and the prevalence of stunting increased by $1.8 \%$ in two years. This showed that no improvement has been recorded in the stunting condition of this age group population from 2013 to 2015 - implying a failure to meet the target reduction in the prevalence of stunting by 2015 as outlined in the Millennium Development Goals. It can be observed that as a child grows older, the degree of magnitude and severity of stunting gets higher. In particular, those aged 12-23 months exhibited higher magnitude and severity of stunting, with more serious condition noted among male children. This finding is similar to the study of Khaldi and Bouguerra (2000), where they found that children grew well until the ages of 5 to 6 months, then growth often faltered with the introduction of inadequate complementary foods and continued to decline until 24 months.

The lower magnitude of stunting within ages 0-11 months and higher magnitude in children 12-23 months could be attributed to infant feeding and complementary feeding practices. As recognised by Shrimpton et al. (2001), the age of 6-24 months in young children is one of the most critical periods of linear growth. This is also the time when the prevalence of stunting is at its peak in developing countries because this period is characterised by high demand for nutrients coupled with limited quality and quantity of complementary foods (Dewy \& Adu-Afarwuah, 2008). These findings are vital for consideration since the first 1000 days of life is the most effective time to prevent stunting. Children suffering from stunting may still be able to reverse that condition to avoid future health and mental problems (Black et al., 2013).

A child being stunted or normal in height was found to be associated with the practice of post-natal check-up, taking an ultrasound, tetanus toxoid vaccine prenatal services, taking folic acid, and single vitamin and multivitamins during pregnancy, diabetic complications during pregnancy, nutritional status of the mother, highest educational attainment, and the duration of lactation of a mother, with the latter having a higher degree of association as compared to the other maternal characteristics. These results can be supported by several previous studies. In a study conducted by Aturupane et al. (2008), it was concluded that maternal education, nutrition knowledge, and post-pregnancy processes contributed to a child's nutritional status. In addition, prenatal services were also found to decrease the odds of birth complications that could affect future child nutrition 
(Turner et al., 1996). Ultrasound is one of the most accessible prenatal services since it allows parents and doctors to detect any abnormalities while the child is still in the womb (Gudex et al., 2006). Tetanus toxoid vaccine is also highly recommended for expecting mothers since the vaccine is known to reduce neonatal mortality (Blencowe et al., 2010). The intake of vitamins during pregnancy is important especially for underweight mothers since micronutrient deficiency can lead to pregnancy complications that can affect the ability to lactate and the growth of the foetus (Yakoob et al., 2010). Furthermore, Piper and Parks (1996) revealed that social status, smoking history of mothers, and parity can all affect the duration of lactation.

In this study, maternal education lowered the likelihood of a child being stunted. Maternal education had been cited by Aturupane et al. (2008) and Babatunde et al. (2011) to be a significant factor in child nutrition in their studies. From the study of Babatunde et al. (2011), a higher level of maternal education would decrease the chances of a child suffering from undernourishment. Children with mothers that have at least a college level of education had a lower chance of being stunted compared to children with mothers that had no formal education. Higher maternal education is often associated with better health practices and nutrition knowledge, which explains the observed decrease in the likelihood of being stunted in all the fitted models. A mother that is either working or still a student also decreases the likelihood of their children being stunted. Boah et al. (2019) suggested that household income has a significant effect on child nutrition, which is related to the employment status of others. Higher educational attainment also suggests better chances of being employed, which may explain why the two maternal characteristics have a positive effect on improving child nutrition.
Several studies have found that mothers being pregnant in the third trimester at the time of survey was an influencing factor to a child's stunting. Blencowe et al. (2010) stated that neonatal and maternal death are greatly reduced by tetanus toxoid vaccination on expecting mothers, which is why it is a staple recommended vaccination during pregnancy. It was also found that mothers having an ultrasound during pregnancy could lower the chance of a child being stunted. Gudex et al. (2006) stated that mothers who only learned of their pregnancy in the second trimester specifically viewed ultrasound as a way to be certain on whether the child was growing normally and void of any abnormalities. They also noted that mothers who had previous abortions or miscarriages hunted for reassurance during ultrasounds.

\section{CONCLUSION AND RECOMMENDATION}

Malnutrition is one of the recurring problems in the Philippines. Stunting, as the most common type of undernutrition among children in the Philippines has been the focus of this study. The results of this study gave further support to the assertion that human capital is necessary to improve child nutrition. A healthy and educated mother would tend to understand the possible longterm effects of malnutrition and why young children need to be well taken care of, thus mothers with enough nutrition knowledge would prevent their child from becoming stunted. According to Martorell and Zongrone (2012), stunting is a cyclical process because a mother who is stunted in childhood tends to have stunted babies. Thus, addressing the problem of stunting early in life is important to break the intergenerational cycle of poverty and reduced human capital that are also associated with stunting. This modelling suggested the strong 
association between formal education of mothers with the height-for-age status of children 0-23 months. Improving the degree of education among women of reproductive age could be a good entry point to reduce the prevalence of stunting in children 0-23 months of age. Also, the Philippines already has numerous health and nutrition programmes. The government should strengthen service delivery among pregnant and lactating women, as well as children ages 0-23 months, to cover the first 1000 days of life. Ensuring a higher coverage rate and accessibility to programmes like micronutrient supplementation, local delivery of maternal and newborn service package, and nutrition education among the target groups may help reduce the magnitude of stunting in the country. Lastly, initiatives towards the promotion of exclusive breastfeeding for the first six months of life, timely introduction of complementary feeding, and continued breastfeeding with a longer lactation period, should be intensified because of their association in lowering the odds of having a 0-23 months child with low height-for-age.

\section{Acknowledgement}

The authors are grateful to the Food and Nutrition Research Institute - Department of Science and Technology, Philippines for the Maternal Health and Nutrition and Infant and Young Child Feeding Surveys (MHN-YCF Surveys), Socio-Economic Survey, and Anthropometric Survey data from the 2015 National Nutrition Updating Survey.

\section{Authors' contributions}

APVH, conceptualised, designed and prepared the manuscript, conducted data analysis and reviewed related articles; NAT, conceptualised, designed and prepared the manuscript; DGCD, conducted review of related literatures. All authors interpreted the results of analysis, read and approved the manuscript.

\section{Conflict of interest}

The authors declared no conflict of interest.

\section{References}

Akoglu H (2018). User's guide to correlation coefficients. Turk J Emerg Med 18(3):91-93.

Altman, DG, \& Bland, JM (1994). Diagnostic tests. 1: Sensitivity and specificity. BMJ (Clinical research ed.), 308(6943), 1552. https://doi. org/10.1136/bmj.308.6943.1552

Aturupane H, A Deolalikar \& D Gunawardene (2008). The determinants of child weight and height in Sri Lanka. UNU-WIDER Research Paper No. 2008/53.

Babatunde RO, Olagunju FI, Fakayode SB \& SolaOjo FE (2011). Prevalence and determinants of malnutrition among under-five children of farming households in Kwara State, Nigeria. $J$ Agric Sci 3(3):173-181

Black RE, Victora CG, Walker SP, Bhutta ZA, Christian P, de Onis M, Ezzati M, GranthamMacGregor S, Katz J, Martorell R and Uauy R (2013). Maternal and child undernutrition and overweight in low-income and middle-income countries. Lancet 382(9890):427-451. https:/ / doi.org/10.1016/S0140-6736(13)60937-X

Blencowe H, Lawn J, Vandelaer J, Roper M \& Cousens S (2010). Tetanus toxoid immunization to reduce mortality from neonatal tetanus. Int $J$ Epidemiol 39(suppl 1):i102-i109.

Boah M, Azupogo F, Amporfro DA \& Abada LA (2019). The epidemiology of undernutrition and its determinants in children under five years in Ghana. PloS One 14(7):e0219665. https://doi. org/10.1371/journal.pone.0219665

de Onis M and Branca F (2016). Childhood stunting: a global perspective. Maternal \& Child Nutrition, 12 Suppl1(Suppl1), 12-26. de Onis, M., \& Branca, F. (2016). Childhood stunting: a global perspective. Maternal \& child nutrition, 12 Suppl 1(Suppl 1), 12-26. https:// doi.org/10.1111/mcn.12231

Dewey KG \& Adu-Afarwuah S (2008). A systematic review of the effectiveness of complementary feeding interventions in developing countries. Matern \& Child Nutrition, 4(Supp 1):24-85. https://doi.org/10.1111/j.17408709.2007.00124.x

Fagerland MW \& Hosmer DW (2012). A generalized Hosmer-Lemeshow goodness-of-fit test for multinomial logistic regression models. Stata $J$ 12(3):447-453.

FNRI-DOST (2015). Philippine Nutrition Facts and Figures 2013: Anthropometric Survey. Food and Nutrition Research Institute- Department of Science and Technology. 
FNRI-DOST (2015). Philippine Nutrition Facts and Figures 2015: Anthropometric Survey. Food and Nutrition Research Institute- Department of Science and Technology.

Gindro, G. (2020). Why you need to test the tests in machine learning. The Data MBA. From https:/ / www.thedatamba.com/post/why-youneed-to-test-the-tests-in-machine-learning [Retrieved June 25, 2020].

Gudex C, Nielsen BL \& Madsen M (2006). Why women want prenatal ultrasound in normal pregnancy. Ultrasound Obstet Gynecol 27(2):145-150.

Khaldi F \& Bouguerra F (2000). Feeding practices, growth, and morbidity in Tunisia. Pediatrics 106(Supplement 4): 1275-1276.

Kandpal E \& McNamara PE (2009). Determinants of nutritional outcomes of children in India: A quantile regression approach. 2009 Annual Meeting, July 26-28, 2009, Milwaukee, Wisconsin 49415, Agricultural and Applied Economics Association.

Martorell R \& Zongrone A (2012). Intergenerational influences on child growth and undernutrition. Pediatr Perinat Epidemiol 26(s1):302-314 doi. org/10.1111/j.1365-3016.2012.01298.x

Ng A (2017). Size of the dev and test sets. [Video format]. From: https://www.coursera.org/ lecture/machine-learning-projects / sizeof-the-dev-and-test-sets-HOby4 [Retrieved November 15 2019].

Piper S \& Parks PL (1996). Predicting the duration of lactation: evidence from a national. Birth 23(1):7-12.

Shrimptom R, Victoria CG, de Onis M, Lima RC, Blössner M \& Clugston G (2001). Worldwide timing of growth faltering: implications for nutritional interventions. Pediatrics 125(3):e473-e480. doi:10.1542/peds.20091519
Turner BJ, McKee LJ, Silverman NS, Hauck WW, Fanning TR \& Markson LE (1996). Prenatal care and birth outcomes of a cohort of HIVinfected women. J Acquir Immune Defic Syndr 12(3):259-267.

UNICEF (2007). Stunting, wasting, and overweight. From https://www unicef org/ progressforchildren/2007n6 index_41505 htm [Retrieved November 15 2019].

UNICEF (2019). The state of the world's children 2019. Children, food and nutrition: Growing well in a changing world. UNICEF, New York.

Yakoob MY, Khan PK and Bhutta ZA (2010). Maternal mineral and vitamin supplementation in pregnancy. Expert Review of Obstetrics \& Gynecology, 5:2, 241-256, DOI: 10.1586/ eog. 10.8

Walker SP, Chang SM, Powell CA, Simonoff E \& Grantham-McGregor SM (2007). Early childhood stunting is associated with poor psychological functioning in late adolescence and effects are reduced by psychosocial stimulation. J Nutr 137(11):2464-2469.

WHO (2008). Training course on child growth assessment. World Health Organization. Geneva, Switzerland.

WHO and UNICEF (2019). Recommendations for data collection, analysis, and reporting on anthropometric indicators in children under 5 years old. World Health Organization and United Nations Children's Fund, Geneva.

Zweig MH \& Campbell G (1993). Receiver-operating characteristic (ROC) plots: a fundamental evaluation tool in clinical medicine. Clin Chem 39(4):561-577. 